\title{
On the Optimal Dynamic Control Strategy of Disruptive Computer Virus
}

\author{
Jichao Bi, ${ }^{1}$ Xiaofan Yang, ${ }^{1}$ Yingbo $\mathrm{Wu},{ }^{1}$ Qingyu Xiong, ${ }^{1}$ \\ Junhao Wen, ${ }^{1}$ and Yuan Yan Tang ${ }^{2}$ \\ ${ }^{1}$ College of Software Engineering, Chongqing University, Chongqing 400044, China \\ ${ }^{2}$ Department of Computer and Information Science, The University of Macau, Macau \\ Correspondence should be addressed to Xiaofan Yang; xfyang1964@gmail.com
}

Received 14 December 2016; Revised 13 February 2017; Accepted 23 February 2017; Published 19 March 2017

Academic Editor: Yong Deng

Copyright (C) 2017 Jichao Bi et al. This is an open access article distributed under the Creative Commons Attribution License, which permits unrestricted use, distribution, and reproduction in any medium, provided the original work is properly cited.

Disruptive computer viruses have inflicted huge economic losses. This paper addresses the development of a cost-effective dynamic control strategy of disruptive viruses. First, the development problem is modeled as an optimal control problem. Second, a criterion for the existence of an optimal control is given. Third, the optimality system is derived. Next, some examples of the optimal dynamic control strategy are presented. Finally, the performance of actual dynamic control strategies is evaluated.

\section{Introduction}

The proliferation of computer networks has brought huge benefits to human society. Meanwhile, it offers a shortcut to spread computer viruses, inflicting large economic losses [1]. Consequently, containing the prevalence of digital viruses has been one of the major concerns in the field of cybersecurity. The spreading dynamics of computer virus has been widely adopted as the standard method for assessing the viral prevalence [2]. Since the seminal work by Kephart and White $[3,4]$, a multitude of computer virus-spreading models, ranging from the population-level models [5-12] and the network-level models [13-17] to the node-level models [1822], have been proposed.

One of the central tasks in cybersecurity is to develop control strategies of computer virus so that, subject to limited budgets, the losses caused by computer infections are minimized [23]. In recent years, the optimal design problem of virus control strategies has been modeled as static optimization problems [24-28]. The optimal static control strategies, however, only apply to the small-timescale situations where the network state keeps unchanged. In the realistic situations where the network state is varying over time, the optimal design problem of virus control strategies can be modeled as dynamic optimal control problems [2933]. The optimal dynamic control strategies outperform their static counterparts, because the former not only are more cost-effective but apply to different timescales.

A disruptive computer virus is defined as a computer virus whose life period consists of two consecutive phases: the latent phase and the disruptive phase. In the latent phase, a disruptive virus staying in a host does not perform any disruptive operations. Rather, the virus tries to infect as many hosts as possible by sending its copies to them. In the disruptive phase, a disruptive virus staying in a host performs a variety of operations that disrupt the host, such as distorting data, deleting data or files, and destroying the operating system. To assess the prevalence of disruptive viruses, a number of virus-spreading models, which are referred to as the Susceptible-Latent-Bursting-Susceptible (SLBS) models, have been suggested [34-38]. The main distinction between the SLBS models and the traditional SEIS models lies in that the latent hosts in the former possess strong infecting capability, whereas the exposed individuals in the latter possess no infecting capability at all. Recently, the basic SLBS models have been extended towards different directions [3943]. At the population-level, Chen et al. [44] developed an optimal dynamic control strategy of disruptive viruses. 
All of the above-mentioned SLBS models are populationlevel; that is, they are based on the assumption that every infected host in the population is equally likely to infect any other susceptible host. These models have two striking defects: (a) the personalized features of different hosts cannot be taken into consideration and (b) the impact of the structure of the virus-propagating network on the viral prevalence cannot be revealed by studying the models. To overcome these defects, Yang et al. [45] presented a node-level SLBS model. In our opinion, optimal dynamic control strategies of disruptive viruses should be developed at the node-level, so as to achieve the best cost-efficiency.

This paper is intended to develop at the node-level an optimal dynamic control strategy of disruptive computer viruses. First, the development problem is modeled as an optimal control problem. Second, a criterion for the existence of an optimal control for the optimal control problem is given. Third, the optimality system for the optimal control problem is presented. Next, some exemplar optimal dynamic control strategies are given. Finally, the difference between the costefficiency of an arbitrary control strategy and that of the optimal dynamic strategy is estimated.

The subsequent materials of this work are organized as follows. Section 2 presents the preliminary knowledge on optimal control theory. Sections 3 and 4 formulate and study the optimal control problem, respectively. Some numerical examples are given in Section 5. Section 6 estimates the aforementioned difference. Finally, Section 7 closes this work.

\section{Fundamental Knowledge}

For fundamental knowledge on optimal control theory, see [46].

Consider the following optimal control problem.

$$
\begin{array}{ll}
\underset{\mathbf{u}(\cdot) \in \mathcal{U}}{\operatorname{Minimize}} \quad J(\mathbf{u}(t)) & \\
& =\int_{0}^{T} F(\mathbf{x}(t), \mathbf{u}(t)) d t \\
\text { subject to } & \frac{d \mathbf{x}(t)}{d t}=\mathbf{f}(\mathbf{x}(t), \mathbf{u}(t)), \\
& \quad 0 \leq t \leq T .
\end{array}
$$

Lemma 1. Problem (P) has an optimal control if the following five conditions hold simultaneously.

$\left(\mathrm{C}_{1}\right) \mathcal{U}$ is closed and convex.

$\left(\mathrm{C}_{2}\right)$ There is $\mathbf{u}(\cdot) \in \mathcal{U}$ such that the adjunctive dynamical system is solvable.

$\left(\mathrm{C}_{3}\right) \mathbf{f}(\mathbf{x}, \mathbf{u})$ is bounded by a linear function in $\mathbf{x}$.

$\left(\mathrm{C}_{4}\right) F(\mathbf{x}, \mathbf{u})$ is convex on $\mathcal{U}$.

$\left(\mathrm{C}_{5}\right) F(\mathbf{x}, \mathbf{u}) \geq c_{1}\|\mathbf{u}\|^{\rho}+c_{2}$ for some vector norm $\|\cdot\|, \rho>$ $1, c_{1}>0$, and $c_{2}$.

\section{Formulation of the Optimal Control Problem}

Consider a population of $N$ hosts (nodes) labelled 1, 2, ., $N$. As with the traditional SLBS models, assume that at any time every node in the population is in one of three possible states: susceptible, latent, and disruptive. Susceptible nodes are those that are not infected with any disruptive computer virus. Latent nodes are those that are infected with some disruptive viruses and all of them are in the latent phase. Disruptive nodes are those that are infected with some disruptive viruses and some of them are in the disruptive phase. Let $X_{i}(t)=0$, 1 , and 2 denote that at time $t$ node $i$ is susceptible, latent, and disruptive, respectively. Let

$$
\begin{aligned}
& S_{i}(t)=\operatorname{Pr}\left\{X_{i}(t)=0\right\}, \\
& L_{i}(t)=\operatorname{Pr}\left\{X_{i}(t)=1\right\}, \\
& B_{i}(t)=\operatorname{Pr}\left\{X_{i}(t)=2\right\} .
\end{aligned}
$$

As $S_{i}(t)+L_{i}(t)+B_{i}(t) \equiv 1(1 \leq i \leq N)$, the vector

$$
\begin{aligned}
& \mathbf{I}(t) \\
& \quad=\left(L_{1}(t), \ldots, L_{N}(t), B_{1}(t), \ldots, B_{N}(t)\right)^{T}
\end{aligned}
$$

probabilistically captures the state of the population at time $t$.

Suppose a dynamic control strategy will be carried out during the time frame $[0, T]$. Let us impose a set of statistical hypotheses as follows.

$\left(\mathrm{H}_{1}\right)$ A susceptible node $i$ is infected by a latent node $j$ at rate $\beta_{L, i j} \geq 0$. Let $\mathbf{A}_{L}=\left(\beta_{L, i j}\right)_{N \times N}$.

$\left(\mathrm{H}_{2}\right)$ A susceptible node $i$ is infected by a disruptive node $j$ at rate $\beta_{B, i j} \geq 0$. Let $\mathbf{A}_{B}=\left(\beta_{B, i j}\right)_{N \times N}$.

$\left(\mathrm{H}_{3}\right)$ Due to the outburst of latent viruses, a latent node $i$ becomes disruptive at rate $\alpha_{i}>0$. Let $\bar{\alpha}=\max _{i} \alpha_{i}$.

$\left(\mathrm{H}_{4}\right)$ Due to the action of new patches, at time $t$ a latent node $i$ becomes susceptible at a controllable rate $\gamma_{L, i}(t) \in L^{2}[0, T]$ and $\gamma_{L} \leq \gamma_{L, i}(t) \leq \overline{\gamma_{L}}$. Hereafter, the symbol $L^{2}[0, T]$ stands for the set of all Lebesgue square integrable functions defined on the interval $[0, T]$. Moreover, the cost needed to achieve the rate at the infinitesimal time interval $[t, t+d t)$ is $p_{i} \gamma_{L, i}^{\theta}(t) d t, p_{i}>0$, and $\theta>0$. This accords with the intuition that the cost increases with $\gamma_{L, i}(t)$.

$\left(\mathrm{H}_{5}\right)$ Due to the action of new patches, at time $t$ a disruptive node $i$ becomes susceptible at a controllable rate $\gamma_{B, i}(t) \in L^{2}[0, T]$ and $\gamma_{B} \leq \gamma_{B, i}(t) \leq \overline{\gamma_{B}}$. Moreover, the cost needed to achieve the rate at the infinitesimal time interval $[t, t+d t)$ is $q_{i} \gamma_{B, i}^{\theta}(t) d t, q_{i}>0$. This conforms to the intuition that the cost increases with $\gamma_{B, i}(t)$.

Figure 1 shows hypotheses $\left(\mathrm{H}_{1}\right)-\left(\mathrm{H}_{5}\right)$ schematically. 


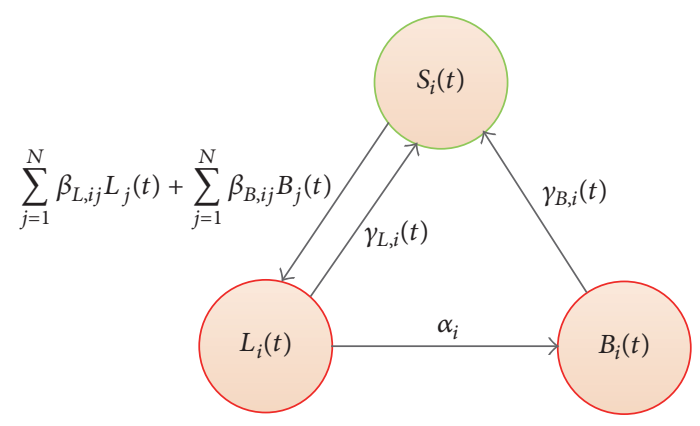

FIGURE 1: Diagram of assumptions $\left(\mathrm{H}_{1}\right)-\left(\mathrm{H}_{5}\right)$.

Let $\Delta t>0$ denote a very small time interval. Hypotheses $\left(\mathrm{H}_{1}\right)-\left(\mathrm{H}_{5}\right)$ imply the following relations.

$$
\begin{gathered}
\operatorname{Pr}\left\{X_{i}(t+\Delta t)=1 \mid X_{i}(t)=0\right\} \\
=\Delta t \sum_{j=1}^{N} \beta_{L, i j} L_{j}(t)+\Delta t \sum_{j=1}^{N} \beta_{B, i j} B_{j}(t)+o(\Delta t), \\
\operatorname{Pr}\left\{X_{i}(t+\Delta t)=2 \mid X_{i}(t)=0\right\}=o(\Delta t), \\
\operatorname{Pr}\left\{X_{i}(t+\Delta t)=2 \mid X_{i}(t)=1\right\}=\alpha_{i} \Delta t+o(\Delta t), \\
\operatorname{Pr}\left\{X_{i}(t+\Delta t)=0 \mid X_{i}(t)=1\right\}=\gamma_{L, i}(t) \Delta t+o(\Delta t), \\
\operatorname{Pr}\left\{X_{i}(t+\Delta t)=0 \mid X_{i}(t)=2\right\}=\gamma_{B, i}(t) \Delta t+o(\Delta t), \\
\operatorname{Pr}\left\{X_{i}(t+\Delta t)=1 \mid X_{i}(t)=2\right\}=o(\Delta t) .
\end{gathered}
$$

As a result, we have

$$
\begin{gathered}
\operatorname{Pr}\left\{X_{i}(t+\Delta t)=0 \mid X_{i}(t)=0\right\} \\
=1-\Delta t \sum_{j=1}^{N} \beta_{L, i j} L_{j}(t) \\
-\Delta t \sum_{j=1}^{N} \beta_{B, i j} B_{j}(t)+o(\Delta t), \\
\operatorname{Pr}\left\{X_{i}(t+\Delta t)=1 \mid X_{i}(t)=1\right\} \\
=1-\alpha_{i} \Delta t-\gamma_{L, i}(t) \Delta t+o(\Delta t), \\
\operatorname{Pr}\left\{X_{i}(t+\Delta t)=2 \mid X_{i}(t)=2\right\} \\
=1-\gamma_{B, i}(t) \Delta t+o(\Delta t) .
\end{gathered}
$$

By the total probability formula, we get

$$
\begin{aligned}
L_{i}(t+ & \Delta t) \\
= & S_{i}(t) \operatorname{Pr}\left\{X_{i}(t+\Delta t)=1 \mid X_{i}(t)=0\right\} \\
& +L_{i}(t) \operatorname{Pr}\left\{X_{i}(t+\Delta t)=1 \mid X_{i}(t)=1\right\} \\
& +B_{i}(t) \operatorname{Pr}\left\{X_{i}(t+\Delta t)=1 \mid X_{i}(t)=2\right\} \\
= & {\left[1-L_{i}(t)-B_{i}(t)\right] \Delta t \sum_{j=1}^{N}\left[\beta_{L, i j} L_{j}(t)+\beta_{B, i j} B_{j}(t)\right] } \\
& +L_{i}(t)-\Delta t\left[\alpha_{i}+\gamma_{L, i}(t)\right] L_{i}(t)+o(\Delta t), \\
B_{i}(t+ & \Delta t) \\
= & S_{i}(t) \operatorname{Pr}\left\{X_{i}(t+\Delta t)=2 \mid X_{i}(t)=0\right\} \\
& +L_{i}(t) \operatorname{Pr}\left\{X_{i}(t+\Delta t)=2 \mid X_{i}(t)=1\right\} \\
& +B_{i}(t) \operatorname{Pr}\left\{X_{i}(t+\Delta t)=2 \mid X_{i}(t)=2\right\} \\
= & \alpha_{i} \Delta t L_{i}(t)+B_{i}(t)-\gamma_{B, i}(t) \Delta t B_{i}(t)+o(\Delta t) .
\end{aligned}
$$

Transposing the terms $L_{i}(t)$ and $B_{i}(t)$ from the right to the left and dividing both sides by $\Delta t$, we get

$$
\begin{aligned}
& \frac{L_{i}(t+\Delta t)-L_{i}(t)}{\Delta t} \\
& =\left[1-L_{i}(t)-B_{i}(t)\right] \sum_{j=1}^{N}\left[\beta_{L, i j} L_{j}(t)+\beta_{B, i j} B_{j}(t)\right] \\
& \quad-\left[\alpha_{i}+\gamma_{L, i}(t)\right] L_{i}(t)+\frac{o(\Delta t)}{\Delta t}, \\
& \frac{B_{i}(t+\Delta t)-B_{i}(t)}{\Delta t}=\alpha_{i} L_{i}(t)-\gamma_{B, i}(t) B_{i}(t)+\frac{o(\Delta t)}{\Delta t} .
\end{aligned}
$$

Letting $\Delta t \rightarrow 0$, we get the following dynamical model.

$$
\begin{aligned}
& \frac{d L_{i}(t)}{d t} \\
& =\left[1-L_{i}(t)-B_{i}(t)\right] \sum_{j=1}^{N}\left[\beta_{L, i j} L_{j}(t)+\beta_{B, i j} B_{j}(t)\right] \\
& \quad-\left[\alpha_{i}+\gamma_{L, i}(t)\right] L_{i}(t), \\
& \frac{d B_{i}(t)}{d t}=\alpha_{i} L_{i}(t)-\gamma_{B, i}(t) B_{i}(t),
\end{aligned}
$$


where $t \geq 0,1 \leq i \leq N$. We refer to the model as the controlled SLBS model, where the control,

$$
\begin{aligned}
& \gamma(t)=\left(\gamma_{L, 1}(t), \ldots, \gamma_{L, N}(t), \gamma_{B, 1}(t), \ldots,\right. \\
& \left.\gamma_{B, N}(t)\right)^{T},
\end{aligned}
$$

stands for a dynamic control strategy of disruptive computer viruses. The admissible set of controls is

$$
\begin{aligned}
\Gamma & =\left\{\gamma(t) \in\left(L^{2}[0, T]\right)^{2 N} \mid \underline{\gamma_{L}} \leq \gamma_{L, i}(t)\right. \\
& \leq \overline{\gamma_{L}}, \underline{\gamma_{B}} \leq \gamma_{B, i}(t) \leq \overline{\gamma_{B}}, 0 \leq t \leq T, 1 \\
& \leq i \leq N\} .
\end{aligned}
$$

$$
\begin{array}{ll}
\underset{\gamma(\cdot) \in \Gamma}{\operatorname{Minimize}} & J(\gamma(\cdot))=\int_{0}^{T} \sum_{i=1}^{N}\left[L_{i}(t)+B_{i}(t)+p_{i} \gamma_{L, i}^{\theta}(t)+q_{i} \gamma_{B, i}^{\theta}(t)\right] d t \\
\text { subject to } & \frac{d \mathbf{I}(t)}{d t}=\mathbf{f}(\mathbf{I}(t), \gamma(t)), \quad 0 \leq t \leq T, \\
& \mathbf{I}(0)=\mathbf{I}_{0} .
\end{array}
$$

A solution to the optimal control problem $\left(\mathrm{P}^{*}\right)$ stands for an optimal dynamic control strategy of disruptive viruses. For convenience, let

$$
\begin{gathered}
F(\mathbf{I}(t), \gamma(t))=\sum_{i=1}^{N}\left[L_{i}(t)+B_{i}(t)\right. \\
\left.+p_{i} \gamma_{L, i}^{\theta}(t)+q_{i} \gamma_{B, i}^{\theta}(t)\right] .
\end{gathered}
$$

\section{A Theoretical Study of the Optimal Control Problem}

In this section, we shall study the optimal control problem $\left(\mathrm{P}^{*}\right)$ presented in the previous section.

4.1. Existence of an Optimal Control. As a solution to the optimal control problem $\left(\mathrm{P}^{*}\right)$ stands for an optimal dynamic control strategy of disruptive viruses, it is critical to show that there is such an optimal control. For that purpose, let us show that the five conditions in Lemma 1 hold true simultaneously.

Lemma 2. The admissible set $\Gamma$ is closed.
Model (7) can be written in matrix notation as

$$
\frac{d \mathbf{I}(t)}{d t}=\mathbf{f}(\mathbf{I}(t), \gamma(t)), \quad 0 \leq t \leq T .
$$

Given a dynamic control strategy $\gamma(\cdot)$. The total loss can be measured by $\int_{0}^{T} \sum_{i=1}^{N}\left[L_{i}(t)+B_{i}(t)\right] d t$, and the total cost can be gauged by $\int_{0}^{T} \sum_{i=1}^{N}\left[p_{i} \gamma_{L, i}^{\theta}(t)+q_{i} \gamma_{B, i}^{\theta}(t)\right] d t$. As a result, the performance of a dynamic control strategy $\gamma(\cdot)$ can be measured by

$$
\begin{gathered}
J(\gamma(\cdot))=\int_{0}^{T} \sum_{i=1}^{N}\left[L_{i}(t)+B_{i}(t)\right. \\
\left.+p_{i} \gamma_{L, i}^{\theta}(t)+q_{i} \gamma_{B, i}^{\theta}(t)\right] d t .
\end{gathered}
$$

Hence, developing an optimal dynamic control strategy of disruptive viruses can be modeled as solving the following optimal control problem.
Proof. Let $\gamma(t)=\left(\gamma_{L, 1}(t), \ldots, \gamma_{L, N}(t), \gamma_{B, 1}(t), \ldots, \gamma_{B, N}(t)\right)^{T}$ be a limit point of $\Gamma$,

$$
\begin{gathered}
\gamma^{(n)}(t)=\left(\gamma_{L, 1}^{(n)}(t), \ldots, \gamma_{L, N}^{(n)}(t), \gamma_{B, 1}^{(n)}(t), \ldots,\right. \\
\left.\gamma_{B, N}^{(n)}(t)\right)^{T}, \quad n=1,2, \ldots,
\end{gathered}
$$

a sequence of points in $\Gamma$ such that

$$
\begin{aligned}
& \left\|\gamma^{(n)}(t)-\gamma(t)\right\|_{2} \\
& =\left[\int_{0}^{T}\left|\gamma^{(n)}(t)-\gamma(t)\right|^{2} d t\right]^{1 / 2}<\frac{1}{n} .
\end{aligned}
$$

The completeness of $\left(L^{2}(0, T)\right)^{2 N}$ implies $\gamma(t) \in L^{2}(0, T)^{2 N}$. Hence, the claim follows from the observation that

$$
\begin{aligned}
& \underline{\gamma_{L}} \leq \gamma_{L, i}(t)=\lim _{n \rightarrow \infty} \gamma_{L, i}^{(n)}(t) \leq \overline{\gamma_{L}}, \\
& \underline{\gamma_{B}} \leq \gamma_{B, i}(t)=\lim _{n \rightarrow \infty} \gamma_{B, i}^{(n)}(t) \leq \overline{\gamma_{B}},
\end{aligned}
$$

$$
1 \leq i \leq N
$$

Lemma 3. The admissible set $\Gamma$ is convex. 
Proof. Let

$$
\begin{aligned}
\gamma^{(1)}(t) & =\left(\gamma_{L, 1}^{(1)}(t), \ldots, \gamma_{L, N}^{(1)}(t), \gamma_{B, 1}^{(1)}(t), \ldots, \gamma_{B, N}^{(1)}(t)\right)^{T} \\
& \in \Gamma \\
\gamma^{(2)}(t) & =\left(\gamma_{L, 1}^{(2)}(t), \ldots, \gamma_{L, N}^{(2)}(t), \gamma_{B, 1}^{(2)}(t), \ldots, \gamma_{B, N}^{(2)}(t)\right)^{T} \\
& \in \Gamma
\end{aligned}
$$

and $0<\kappa<1$. As $\left(L^{2}[0, T]\right)^{2 N}$ is a real vector space, we get

$$
\begin{gathered}
(1-\kappa) \gamma^{(1)}(t)+\kappa \gamma^{(2)}(t) \\
\in\left(L^{2}[0, T]\right)^{2 N} .
\end{gathered}
$$

So, the claim follows from the observation that

$$
\begin{aligned}
& \underline{\gamma_{L}} \leq(1-\kappa) \gamma_{L, i}^{(1)}(t)+\kappa \gamma_{L, i}^{(2)}(t) \leq \overline{\gamma_{L}} \\
& \underline{\gamma_{B}} \leq(1-\kappa) \gamma_{B, i}^{(1)}(t)+\kappa \gamma_{B, i}^{(2)}(t) \leq \overline{\gamma_{B}},
\end{aligned}
$$

$$
1 \leq i \leq N \text {. }
$$

Lemma 4. There is $\gamma \in \Gamma$ such that model (7) is solvable.
Proof. Substituting $\gamma(t) \equiv \bar{\gamma}=\left(\overline{\gamma_{L}}, \ldots, \overline{\gamma_{L}}, \overline{\gamma_{B}}, \ldots, \overline{\gamma_{B}}\right)^{T}$ into model (7), we get

$$
\frac{d \mathbf{I}(t)}{d t}=\mathbf{f}(\mathbf{I}(t), \bar{\gamma}), \quad 0 \leq t \leq T .
$$

As $\mathbf{f}(\mathbf{I}, \bar{\gamma})$ is continuously differentiable, the claim follows from the Continuation Theorem for Differential Systems [47].

Lemma 5. $\mathbf{f}(\mathbf{I}, \gamma)$ is bounded by a linear function in $\mathbf{I}$.

Proof. The claim follows from the observation that, for $i=$ $1,2, \ldots, N$,

$$
\begin{gathered}
\left(1-L_{i}-B_{i}\right) \sum_{j=1}^{N}\left(\beta_{L, i j} L_{j}+\beta_{B, i j} B_{j}\right) \\
-\left(\alpha_{i}+\gamma_{L, i}\right) L_{i} \leq \sum_{j=1}^{N} \beta_{L, i j} L_{j} \\
+\sum_{j=1}^{N} \beta_{B, i j} B_{j}-\left(\alpha_{i}+\underline{\gamma_{L}}\right) L_{i} \\
\alpha_{i} L_{i}-\gamma_{B, i} B_{i} \leq \alpha_{i} L_{i}-\underline{\gamma_{B}} B_{i} .
\end{gathered}
$$

Lemma 6. $F(\mathbf{I}, \gamma)$ is convex on $\Gamma$ if $\theta \geq 1$.

Proof. The Hessian of $F$ with respect to $\gamma$,

$$
\begin{aligned}
& {\left[\begin{array}{cccccc}
\frac{\partial^{2} F}{\partial \gamma_{L, 1}^{2}} & \cdots & \frac{\partial^{2} F}{\partial \gamma_{L, 1} \partial \gamma_{L, N}} & \frac{\partial^{2} F}{\partial \gamma_{L, 1} \partial \gamma_{B, 1}} & \cdots & \frac{\partial^{2} F}{\partial \gamma_{L, 1} \partial \gamma_{B, N}} \\
\vdots & \ddots & \vdots & \vdots & \ddots & \vdots \\
\frac{\partial^{2} F}{\partial \gamma_{L, N} \partial \gamma_{L, 1}} & \cdots & \frac{\partial^{2} F}{\partial \gamma_{L, N}^{2}} & \frac{\partial^{2} F}{\partial \gamma_{L, N} \partial \gamma_{B, 1}} & \cdots & \frac{\partial^{2} F}{\partial \gamma_{L, N} \partial \gamma_{B, N}} \\
\frac{\partial^{2} F}{\partial \gamma_{B, 1} \partial \gamma_{L, 1}} & \cdots & \frac{\partial^{2} F}{\partial \gamma_{B, 1} \partial \gamma_{L, N}} & \frac{\partial^{2} F}{\partial \gamma_{B, 1}^{2}} & \cdots & \frac{\partial^{2} F}{\partial \gamma_{B, 1} \partial \gamma_{B, N}} \\
\vdots & \ddots & \vdots & \vdots & \ddots & \vdots \\
\frac{\partial^{2} F}{\partial \gamma_{B, N} \partial \gamma_{L, 1}} & \cdots & \frac{\partial^{2} F}{\partial \gamma_{B, N} \partial \gamma_{L, N}} & \frac{\partial^{2} F}{\partial \gamma_{B, N} \partial \gamma_{B, 1}} & \cdots & \frac{\partial^{2} F}{\partial \gamma_{B, N}^{2}}
\end{array}\right]} \\
& =\theta(\theta-1)\left[\begin{array}{cccccc}
p_{1} \gamma_{L, 1}^{\theta-2} & \cdots & 0 & 0 & \cdots & 0 \\
\vdots & \ddots & \vdots & \vdots & \ddots & \vdots \\
0 & \cdots & p_{N} \gamma_{L, N}^{\theta-2} & 0 & \cdots & 0 \\
0 & \cdots & 0 & q_{1} \gamma_{B, 1}^{\theta-2} & \cdots & 0 \\
\vdots & \ddots & \vdots & \vdots & \ddots & \vdots \\
0 & \cdots & 0 & 0 & \cdots & q_{N} \gamma_{B, N}^{\theta-2}
\end{array}\right],
\end{aligned}
$$


is always positive semidefinite. This implies the convexity of F.

Lemma 7. $F(\mathbf{I}, \gamma) \geq \min _{i}\left\{c_{i}, d_{i}\right\}\|\mathbf{I}\|_{\theta}^{\theta}$, where $\|\cdot\|_{\theta}$ stands for the $\theta$-norm of vectors.

Proof. We have

$$
\begin{aligned}
F(\mathbf{I}, \gamma) & =\sum_{i=1}^{N}\left(L_{i}+B_{i}+p_{i} \gamma_{L, i}^{\theta}+q_{i} \gamma_{B, i}^{\theta}\right) \\
& \geq \min _{1 \leq i \leq N}\left\{p_{i}, q_{i}\right\} \sum_{i=1}^{N}\left(\gamma_{L, i}^{\theta}+\gamma_{B, i}^{\theta}\right) \\
& =\min _{1 \leq i \leq N}\left\{p_{i}, q_{i}\right\}\|\mathbf{I}\|_{\theta}^{\theta} .
\end{aligned}
$$

We are ready to present the main result of this subsection.

Theorem 8. Problem $\left(\mathrm{P}^{*}\right)$ has an optimal control if $\theta>1$.

Proof. Lemmas 2-7 show that the five conditions in Lemma 1 are all met. Hence, the existence of an optimal control follows from Lemma 1.

4.2. The Optimality System. As the optimality system for the optimal control problem $\left(\mathrm{P}^{*}\right)$ offers a method for numerically solving the problem, it is critical to determine the optimality system. For that purpose, consider the corresponding Hamiltonian

$$
\begin{aligned}
& H(\mathbf{I}(t), \gamma(t), \lambda(t)) \\
& \quad=\sum_{i=1}^{N}\left[L_{i}(t)+B_{i}(t)+p_{i} \gamma_{L, i}^{\theta}(t)+q_{i} \gamma_{2 i}^{\theta}(t)\right] \\
& +\sum_{i=1}^{N} \lambda_{L, i}(t) \\
& \quad \cdot\left\{1-L_{i}(t)-B_{i}(t)\right] \sum_{j=1}^{N}\left[\beta_{L, i j} L_{j}(t)+\beta_{B, i j} B_{j}(t)\right] \\
& \left.\quad-\left[\alpha_{i}+\gamma_{L, i}(t)\right] L_{i}(t)\right\}+\sum_{i=1}^{N} \lambda_{B, i}(t)\left[\alpha_{i} L_{i}(t)\right. \\
& \left.\quad-\gamma_{B, i}(t) B_{i}(t)\right],
\end{aligned}
$$

where $\lambda(\cdot)=\left(\lambda_{L, 1}(\cdot), \ldots, \lambda_{L, N}(\cdot), \lambda_{B, 1}(\cdot), \ldots, \lambda_{B, N}(\cdot)\right)^{T}$ is the adjoint.

Theorem 9. Suppose $\gamma^{*}(\cdot)$ is an optimal control for problem $\left(\mathrm{P}^{*}\right)$ with $\theta>1 ; \mathbf{I}^{*}(\cdot)$ is the solution to the controlled SLBS model with $\gamma(\cdot)=\gamma^{*}(\cdot)$. Then, there exists $\lambda^{*}(\cdot)$ with $\lambda^{*}(T)=\mathbf{0}$ such that

$$
\frac{d \lambda_{L, i}^{*}(t)}{d t}=-1+\lambda_{L, i}^{*}(t)\left\{\alpha_{i}+\gamma_{L, i}^{*}(t)\right.
$$

$$
\begin{aligned}
& \left.+\sum_{j=1}^{N}\left[\beta_{L, i j} L_{j}^{*}(t)+\beta_{B, i j} B_{j}^{*}(t)\right]\right\} \\
& -\sum_{j=1}^{N} \beta_{L, j i}\left[1-L_{j}^{*}(t)-B_{j}^{*}(t)\right] \lambda_{L, j}^{*}(t) \\
& -\alpha_{i} \lambda_{B, i}^{*}(t), \\
& \frac{d \lambda_{B, i}^{*}(t)}{d t}=-1+\gamma_{B, i}^{*}(t) \lambda_{B, i}^{*}(t)+\lambda_{L, i}^{*}(t) \\
& . \sum_{j=1}^{N}\left[\beta_{L, i j} L_{j}^{*}(t)+\beta_{B, i j} B_{j}^{*}(t)\right] \\
& -\sum_{j} \beta_{B, j i}\left[1-L_{j}^{*}(t)-B_{j}^{*}(t)\right] \lambda_{L, j}^{*}(t),
\end{aligned}
$$

$\gamma_{L, i}^{*}(t)$

$$
=\max \left\{\operatorname { m i n } \left\{\left[\frac{\lambda_{L, i}^{*}(t) L_{i}^{*}(t)}{\theta p_{i}}\right]^{1 /(\theta-1)},\right.\right.
$$

$$
\left.\left.\overline{\gamma_{L}}\right\}, \underline{\gamma_{L}}\right\}
$$

$\gamma_{B, i}^{*}(t)$

$$
\begin{aligned}
& =\max \left\{\operatorname { m i n } \left\{\left[\frac{\lambda_{B, i}^{*}(t) B_{i}^{*}(t)}{\theta q_{i}}\right]^{1 /(\theta-1)},\right.\right. \\
& \left.\left.\overline{\gamma_{B}}\right\}, \underline{\gamma_{B}}\right\},
\end{aligned}
$$

where $0 \leq t \leq T$ and $1 \leq i \leq N$.

Proof. According to the Pontryagin Minimum Principle [26], there exists $\lambda^{*}(t)$ such that

$$
\begin{aligned}
& \frac{d \lambda_{L, i}^{*}(t)}{d t}=-\frac{\partial H\left(\mathbf{I}^{*}(t), \gamma^{*}(t), \lambda^{*}(t)\right)}{\partial L_{i}}, \\
& 0 \leq t \leq T, 1 \leq i \leq N,
\end{aligned}
$$




$$
\begin{array}{r}
\frac{d \lambda_{B, i}^{*}(t)}{d t}=-\frac{\partial H\left(\mathbf{I}^{*}(t), \gamma^{*}(t), \lambda^{*}(t)\right)}{\partial B_{i}}, \\
0 \leq t \leq T, 1 \leq i \leq N .
\end{array}
$$

Thus, the first $2 \mathrm{~N}$ equations in the claim follow by direct calculations. As the terminal cost is unspecified and the final state is free, the transversality condition $\lambda^{*}(T)=\mathbf{0}$ holds. By using the optimality condition

$$
\begin{aligned}
\gamma^{*} & (t) \\
& =\underset{\gamma(t) \in \Gamma}{\arg \min _{\boldsymbol{N}} H}\left(\mathbf{I}^{*}(t), \gamma(t), \lambda^{*}(t)\right),
\end{aligned}
$$

we get (a) either

$$
\begin{aligned}
& \frac{\partial H\left(\mathbf{I}^{*}(t), \gamma^{*}(t), \lambda^{*}(t)\right)}{\partial \gamma_{L, i}} \\
& =\theta p_{i}\left(\gamma_{L, i}^{*}(t)\right)^{\theta-1}-\lambda_{L, i}^{*}(t) L_{i}^{*}(t)=0
\end{aligned}
$$

or $\gamma_{L, i}^{*}(t)=\underline{\gamma_{L}}$ or $\gamma_{L, i}^{*}(t)=\overline{\gamma_{L}}$ and (b) either

$$
\begin{aligned}
& \frac{\partial H\left(\mathbf{I}^{*}(t), \gamma^{*}(t), \lambda^{*}(t)\right)}{\partial \gamma_{B, i}} \\
& =\theta q_{i}\left(\gamma_{B, i}^{*}(t)\right)^{\theta-1}-\lambda_{B, i}^{*}(t) B_{i}^{*}(t)=0
\end{aligned}
$$

or $\gamma_{B, i}^{*}(t)=\underline{\gamma_{B}}$ or $\gamma_{B, i}^{*}(t)=\overline{\gamma_{B}}$. So, the last $2 N$ equations in the claim follow.

By combining the above discussions, we get the optimality system for problem $\left(\mathrm{P}^{*}\right)$ with $\theta>1$ as follows.

$$
\begin{aligned}
& \frac{d L_{i}(t)}{d t}=\left[1-L_{i}(t)-B_{i}(t)\right] \\
& \cdot \sum_{j=1}^{N}\left[\beta_{L, i j} L_{j}(t)+\beta_{B, i j} B_{j}(t)\right]-\left[\alpha_{i}\right. \\
& \left.+\gamma_{L, i}(t)\right] L_{i}(t), \\
& \frac{d B_{i}(t)}{d t}=\alpha_{i} L_{i}(t)-\gamma_{B, i}(t) B_{i}(t), \\
& \frac{d \lambda_{L, i}(t)}{d t}=-1+\lambda_{L, i}(t)\left\{\alpha_{i}+\gamma_{L, i}(t)\right. \\
& \left.+\sum_{j=1}^{N}\left[\beta_{L, i j} L_{j}(t)+\beta_{B, i j} B_{j}(t)\right]\right\}
\end{aligned}
$$

$$
\begin{aligned}
& -\sum_{j=1}^{N} \beta_{L, j i}\left[1-L_{j}(t)-B_{j}(t)\right] \lambda_{L, j}(t) \\
& -\alpha_{i} \lambda_{B, i}(t), \\
& \frac{d \lambda_{B, i}(t)}{d t}=-1+\gamma_{B, i}(t) \lambda_{B, i}(t)+\lambda_{L, i}(t) \\
& \cdot \sum_{j=1}^{N}\left[\beta_{L, i j} L_{j}(t)+\beta_{B, i j} B_{j}(t)\right] \\
& -\sum_{j=1}^{N} \beta_{B, j i}\left[1-L_{j}(t)-B_{j}(t)\right] \lambda_{L, j}(t),
\end{aligned}
$$$$
\gamma_{L, i}(t)
$$$$
=\max \left\{\operatorname { m i n } \left\{\left[\frac{\lambda_{L, i}(t) L_{i}(t)}{\theta p_{i}}\right]^{1 /(\theta-1)},\right.\right.
$$$$
\left.\left.\overline{\gamma_{L}}\right\}, \underline{\gamma_{L}}\right\},
$$

$\gamma_{B, i}(t)$

$$
\begin{aligned}
& =\max \left\{\operatorname { m i n } \left\{\left[\frac{\lambda_{B, i}(t) B_{i}(t)}{\theta q_{i}}\right]^{1 /(\theta-1)},\right.\right. \\
& \left.\left.\overline{\gamma_{B}}\right\}, \underline{\gamma_{B}}\right\},
\end{aligned}
$$

where $\mathbf{I}(0)=\mathbf{I}_{0}, \lambda(T)=\mathbf{0}, 0 \leq t \leq T, 1 \leq i \leq N$.

By applying the forward-backward Euler scheme to the optimality system, we can obtain the numerical solution to the optimal control problem $\left(\mathrm{P}^{*}\right)$, that is, an optimal dynamic control strategy of disruptive viruses.

\section{Numerical Examples}

This section gives some examples of the optimal dynamic control strategy of disruptive computer viruses. Given a dynamic control strategy $\gamma(t)$. Define the average control (AC) function, the average cumulative loss (ACL) function, the average cumulative cost (ACC) function, and the average cumulative performance (ACP) function as follows.

$$
\mathrm{AC}(t)=\frac{1}{N} \sum_{i=1}^{N}\left[\gamma_{L, i}(t)+\gamma_{B, i}(t)\right],
$$




$$
\begin{aligned}
& \operatorname{ACL}(t)=\frac{1}{N} \sum_{i=1}^{N} \int_{0}^{t}\left[L_{i}(s)+B_{i}(s)\right] d s, \\
& \operatorname{ACC}(t)=\frac{1}{N} \sum_{i=1}^{N} \int_{0}^{t}\left[p_{i} \gamma_{L, i}^{\theta}(s)\right. \\
& \left.+q_{i} \gamma_{B, i}^{\theta}(s)\right] d s, \quad 0 \leq t \leq T, \\
& \operatorname{ACP}(t)=\frac{1}{N} \sum_{i=1}^{N} \int_{0}^{t}\left[L_{i}(s)+B_{i}(s)\right. \\
& \left.+p_{i} \gamma_{L, i}^{\theta}(s)+q_{i} \gamma_{B, i}^{\theta}(s)\right] d s,
\end{aligned}
$$

These functions form an evaluation criterion of dynamic control strategies of disruptive viruses.

5.1. Scale-Free Network. Scale-free networks are a large class of networks having widespread applications. For our purpose, generate a scale-free network $G$ with $N=100$ nodes using the Barabasi-Albert method [48].

Example 10. Consider an optimal control problem $\left(\mathrm{P}^{*}\right)$ on the virus-spreading network $G$, where the parameters and the initial conditions are set as follows.
(a) $T=200, \theta=2, \underline{\gamma_{L}}=0, \overline{\gamma_{L}}=0.2, \underline{\gamma_{B}}=0.1$, and $\overline{\gamma_{B}}=0.3$.
(b) $\beta_{L, i j}=0.005$ and $\beta_{B, i j}=0.001,(i, j) \in E(G)$.
(c) $\alpha_{i}=0.1$ and $p_{i}=q_{i}=1, i \in V(G)$.
(d) $L_{i}(0)=0.1$ and $B_{i}(0)=0,1 \leq i \leq N$.

For the optimal dynamic control strategy to the optimal control problem and some static control strategies, the $\mathrm{AC}$ functions, the ACL functions, the ACC functions, and the ACP function are shown in Figure 2.

5.2. Small-World Network. Small-world networks are another large class of networks having widespread applications. For our purpose, generate a small-world network $G$ with $N=100$ nodes using the Watts-Strogatz method [49].

Example 11. Consider an optimal control problem $\left(\mathrm{P}^{*}\right)$ on the virus-spreading network $G$, where the parameters and the initial conditions are set as follows.

(a) $T=200, \theta=2, \underline{\gamma_{L}}=0, \overline{\gamma_{L}}=0.2, \underline{\gamma_{B}}=0.1$, and $\overline{\gamma_{B}}=$ 0.3 .

(b) $\beta_{L, i j}=0.005$ and $\beta_{B, i j}=0.001,(i, j) \in E(G)$.

(c) $\alpha_{i}=0.1$ and $p_{i}=q_{i}=1, i \in V(G)$.

(d) $L_{i}(0)=0.1$ and $B_{i}(0)=0,1 \leq i \leq N$.
For the optimal dynamic control strategy to the optimal control problem and some static control strategies, the AC functions, the ACL functions, the ACC functions, and the ACP function are shown in Figure 3.

5.3. Realistic Network. Consider a network $G$ with $N=300$ nodes cut out from the database of Stanford University [50].

Example 12. Consider an optimal control problem $\left(\mathrm{P}^{*}\right)$ on the virus-spreading network $G$, where the parameters and the initial conditions are set as follows.

(a) $T=200, \theta=2, \underline{\gamma_{L}}=0, \overline{\gamma_{L}}=0.2, \underline{\gamma_{B}}=0.1$, and $\overline{\gamma_{B}}=$ 0.3

(b) $\beta_{L, i j}=0.005$ and $\beta_{B, i j}=0.001,(i, j) \in E(G)$.

(c) $\alpha_{i}=0.1$ and $p_{i}=q_{i}=1, i \in V(G)$.

(d) $L_{i}(0)=0.1$ and $B_{i}(0)=0,1 \leq i \leq N$.

For the optimal dynamic control strategy to the optimal control problem and some static control strategies, the AC functions, the ACL functions, the ACC functions, and the ACP function are shown in Figure 4.

\section{Performance Evaluation}

The previous discussions manifest that if the parameters in the optimal control problem $\left(\mathrm{P}^{*}\right)$ are all available, then an optimal dynamic control strategy can be obtained by numerically solving the optimality system. In realistic scenarios, however, some of these parameters might be unavailable. In such situations, it is necessary to estimate the performance of an actual dynamical control strategy in comparison with that of the optimal dynamical control strategy. Now let us present such an estimation.

Theorem 13. Consider the optimal control problem $\left(\mathrm{P}^{*}\right)$. Let $\gamma^{*}(\cdot)$ be the optimal dynamic control strategy, $\gamma(\cdot)$ an arbitrary dynamic control strategy. Then,

$$
\begin{aligned}
& \left|J(\gamma(\cdot))-J\left(\gamma^{*}(\cdot)\right)\right| \\
& \leq \frac{2 N c_{1}}{c_{2}}\left(e^{c_{2} T}-1-c_{2} T-\frac{c_{2}^{2} T^{2}}{2}\right) \\
& \quad+\sum_{i=1}^{N} p_{i} \int_{0}^{T}\left|\gamma_{L, i}^{\theta}(t)-\gamma_{L, i}^{* \theta}(t)\right| d t \\
& \quad+\sum_{i=1}^{N} q_{i} \int_{0}^{T}\left|\gamma_{B, i}^{\theta}(t)-\gamma_{B, i}^{* \theta}(t)\right| d t
\end{aligned}
$$




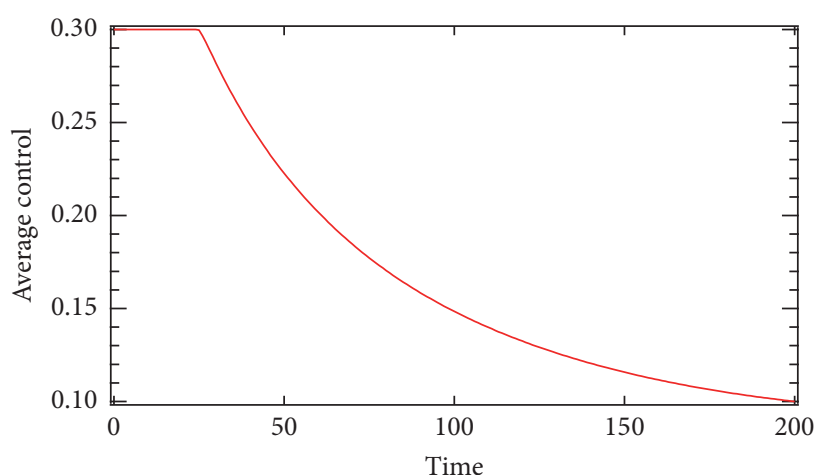

_ Optimal control

(a)

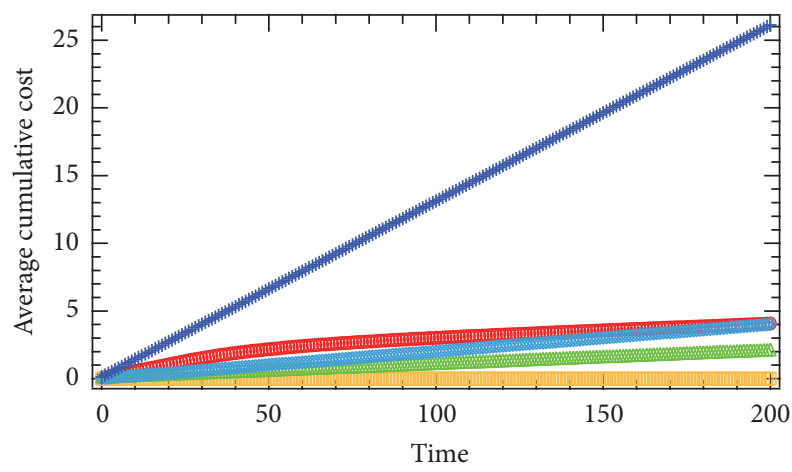

- Optimal control

$\square \gamma_{L}=0.0, \gamma_{B}=0.0$

$\Delta \gamma_{L}=0.0, \gamma_{B}=0.1$

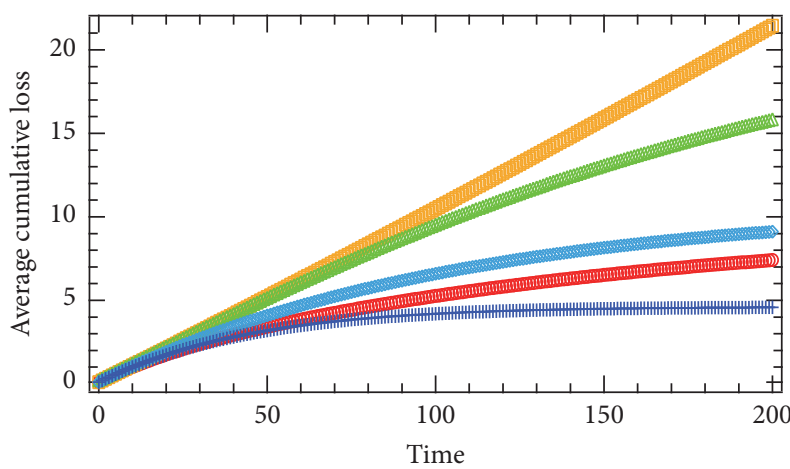

- Optimal control

$\square \gamma_{L}=0.0, \gamma_{B}=0.0$

$\triangle \gamma_{L}=0.0, \gamma_{B}=0.1$

$\diamond \gamma_{L}=0.1, \gamma_{B}=0.1$

$+\gamma_{L}=0.2, \gamma_{B}=0.3$

(b)

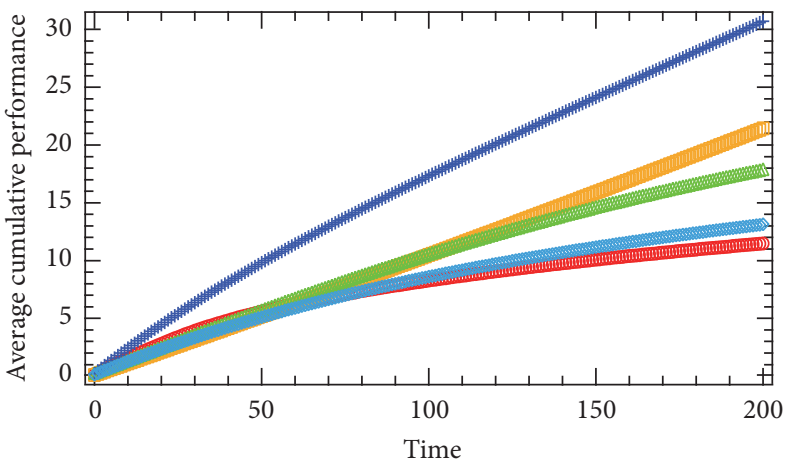

- Optimal control

$\square \gamma_{L}=0.0, \gamma_{B}=0.0$

$\triangle \gamma_{L}=0.0, \gamma_{B}=0.1$ $\diamond \gamma_{L}=0.1, \gamma_{B}=0.1$

$+\gamma_{L}=0.2, \gamma_{B}=0.3$

(d)

FIgure 2: (a) The AC functions, (b) the ACL functions, (c) the ACC functions, and (d) the ACP functions for the optimal dynamic control strategies in Example 10.

where

$$
\begin{aligned}
c_{1} & =\max \left\{2 \max _{1 \leq i \leq N}\left\{\sum_{j=1}^{N}\left|\beta_{L, i j}\right|\right\}\right. \\
& +2 \max _{1 \leq i \leq N}\left\{\sum_{j=1}^{N}\left|\beta_{B, i j}\right|\right\}+\overline{\gamma_{L}}-\underline{\gamma_{L}}, \overline{\gamma_{B}} \\
& \left.-\underline{\gamma_{B}}\right\}, \quad \\
c_{2} & =2 \max _{1 \leq i \leq N}\left\{\sum_{j=1}^{N}\left|\beta_{L, i j}\right|\right\}+\bar{\alpha}+\overline{\gamma_{L}} \\
& +\max \left\{2 \max _{1 \leq i \leq N}\left\{\sum_{j=1}^{N}\left|\beta_{B, i j}\right|\right\}, \overline{\gamma_{B}}\right\} .
\end{aligned}
$$

Proof. Let $\|\cdot\|$ denote the $\boldsymbol{\infty}$-norm. Let $\mathbf{I}^{*}(\cdot)=\left(\mathbf{L}^{*}(\cdot)^{T}\right.$, $\left.\mathbf{B}^{*}(\cdot)^{T}\right)^{T}$ denote the solution to the SLBS model with control $\gamma^{*}(t)$ and $\mathbf{I}(\cdot)=\left(\mathbf{L}(\cdot)^{T}, \mathbf{B}(\cdot)^{T}\right)^{T}$ the solution to the SLBS model with control $\gamma(\cdot)$. As

$$
\begin{aligned}
& \mathbf{L}(t)=\mathbf{L}_{0}^{*}+\int_{0}^{t} \operatorname{diag}\left(1-B_{i}(s)-L_{i}(s)\right) \\
& \cdot \mathbf{A}_{L} \mathbf{L}(s) d s \\
& +\int_{0}^{t} \operatorname{diag}\left(1-B_{i}(s)-L_{i}(s)\right) \\
& \cdot \mathbf{A}_{B} \mathbf{B}(s) d s \\
& -\int_{0}^{t} \operatorname{diag}\left(\alpha_{i}+\gamma_{L, i}(s)\right) \mathbf{L}(s) d s, \\
& \mathbf{L}^{*}(t)=\mathbf{L}_{0}^{*} \\
& \quad+\int_{0}^{t} \operatorname{diag}\left(1-B_{i}^{*}(s)-L_{i}^{*}(s)\right) \\
& \cdot \mathbf{A}_{L} \mathbf{L}^{*}(s) d s
\end{aligned}
$$




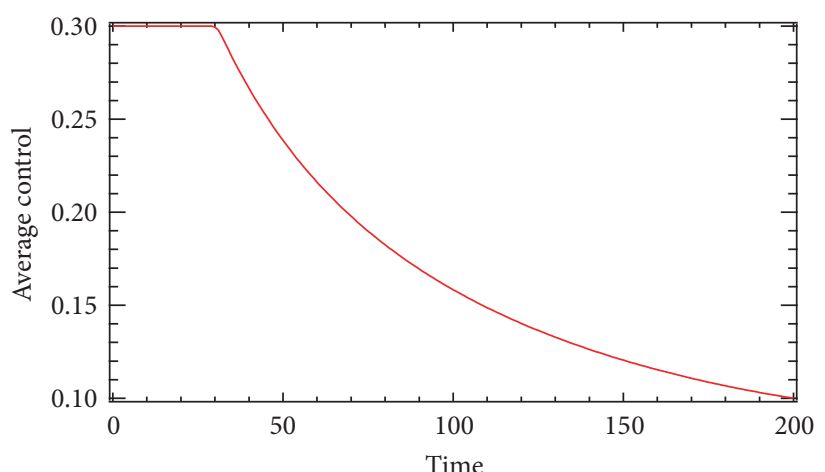

_ Optimal control

(a)

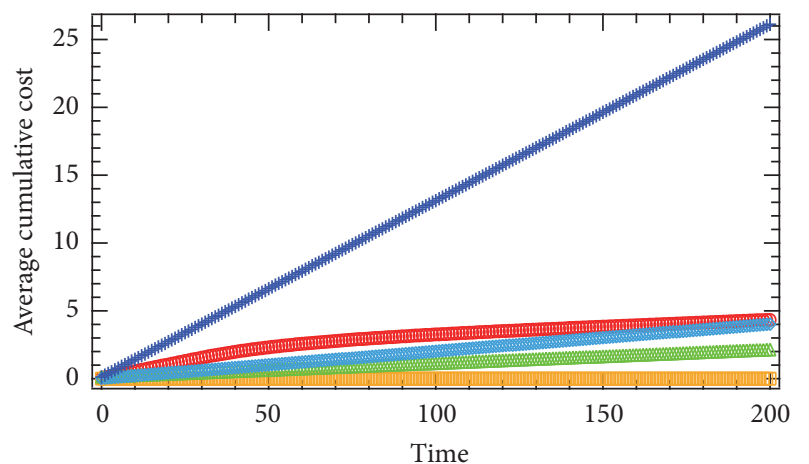

$\begin{array}{ll}\circ & \text { Optimal control } \\ \square & \gamma_{L}=0.0, \gamma_{B}=0.0 \\ \triangle & \gamma_{L}=0.0, \gamma_{B}=0.1\end{array}$

$\diamond \gamma_{L}=0.1, \gamma_{B}=0.1$

$+\gamma_{L}=0.2, \gamma_{B}=0.3$

(c)

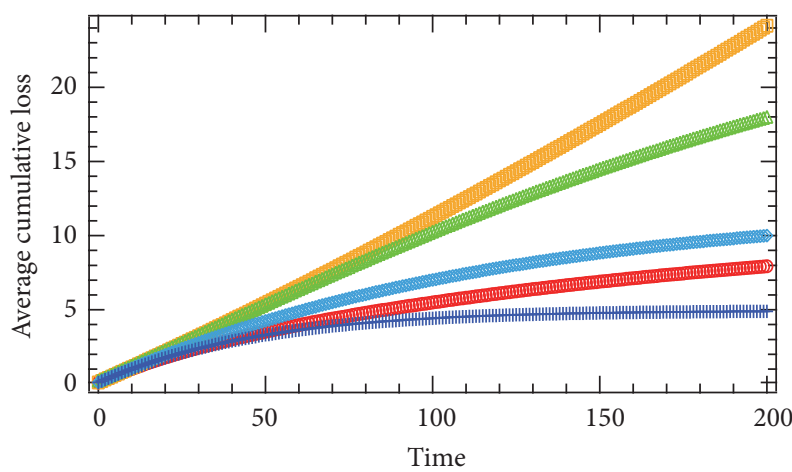

○ Optimal control $\quad \diamond \gamma_{L}=0.1, \gamma_{B}=0.1$

$\square \gamma_{L}=0.0, \gamma_{B}=0.0$

$\triangle \gamma_{L}=0.0, \gamma_{B}=0.1$

$+\gamma_{L}=0.2, \gamma_{B}=0.3$

(b)

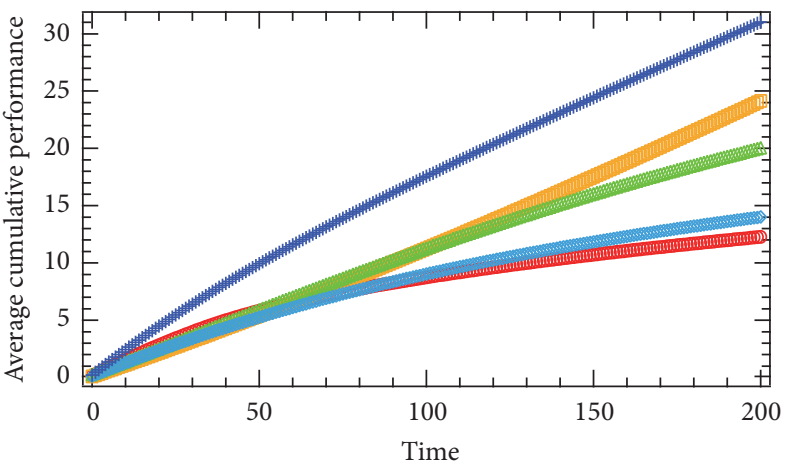

$$
\begin{array}{lll}
\circ \text { Optimal control } & \diamond \gamma_{L}=0.1, \gamma_{B}=0.1 \\
\square \gamma_{L}=0.0, \gamma_{B}=0.0 & +\gamma_{L}=0.2, \gamma_{B}=0.3
\end{array}
$$

$\Delta \gamma_{L}=0.0, \gamma_{B}=0.1$

(d)

Figure 3: (a) The AC functions, (b) the ACL functions, (c) the ACC functions, and (d) the ACP functions for the optimal dynamic control strategies in Example 11.

$$
\begin{aligned}
& +\int_{0}^{t} \operatorname{diag}\left(1-B_{i}^{*}(s)-L_{i}^{*}(s)\right) \\
& \cdot \mathbf{A}_{B} \mathbf{B}^{*}(s) d s \\
& -\int_{0}^{t} \operatorname{diag}\left(\alpha_{i}+\gamma_{L, i}^{*}(s)\right) \mathbf{L}^{*}(s) d s,
\end{aligned}
$$

we get

$$
\begin{aligned}
\mathbf{L}(t) & -\mathbf{L}^{*}(t)=\int_{0}^{t} \operatorname{diag}\left(1-B_{i}(s)-L_{i}(s)\right) \\
\cdot & \mathbf{A}_{L}\left[\mathbf{L}(s)-\mathbf{L}^{*}(s)\right] d s \\
& +\int_{0}^{t} \operatorname{diag}\left(1-B_{i}(s)-L_{i}(s)\right) \\
& \cdot \mathbf{A}_{B}\left[\mathbf{B}(s)-\mathbf{B}^{*}(s)\right] d s \\
& -\int_{0}^{t} \operatorname{diag}\left(L_{i}(s)-L_{i}^{*}(s)+B_{i}(s)-B_{i}^{*}(s)\right) \\
& \cdot \mathbf{A}_{L} \mathbf{L}^{*}(s) d s
\end{aligned}
$$

$$
\begin{aligned}
& -\int_{0}^{t} \operatorname{diag}\left(L_{i}(s)-L_{i}^{*}(s)+B_{i}(s)-B_{i}^{*}(s)\right) \\
& \cdot \mathbf{A}_{B} \mathbf{B}^{*}(s) d s-\int_{0}^{t} \operatorname{diag}\left(\alpha_{i}+\gamma_{L, i}(s)\right) \\
& \cdot\left[\mathbf{L}(s)-\mathbf{L}^{*}(s)\right] d s-\int_{0}^{t} \operatorname{diag}\left(\gamma_{L, i}(s)-\gamma_{L, i}^{*}(s)\right)
\end{aligned}
$$$$
\cdot \mathbf{L}^{*}(s) d s
$$

So,

$$
\begin{aligned}
& \left\|\mathbf{L}(t)-\mathbf{L}^{*}(t)\right\| \leq\left\|\mathbf{A}_{L}\right\| \int_{0}^{t}\left\|\operatorname{diag}\left(1-B_{i}(s)-L_{i}(s)\right)\right\| \\
& \cdot\left\|\mathbf{L}(s)-\mathbf{L}^{*}(s)\right\| d s+\left\|\mathbf{A}_{B}\right\| \\
& \cdot \int_{0}^{t}\left\|\operatorname{diag}\left(1-B_{i}(s)-L_{i}(s)\right)\right\| \\
& \cdot\left\|\mathbf{B}(s)-\mathbf{B}^{*}(s)\right\| d s+\left\|\mathbf{A}_{L}\right\|
\end{aligned}
$$




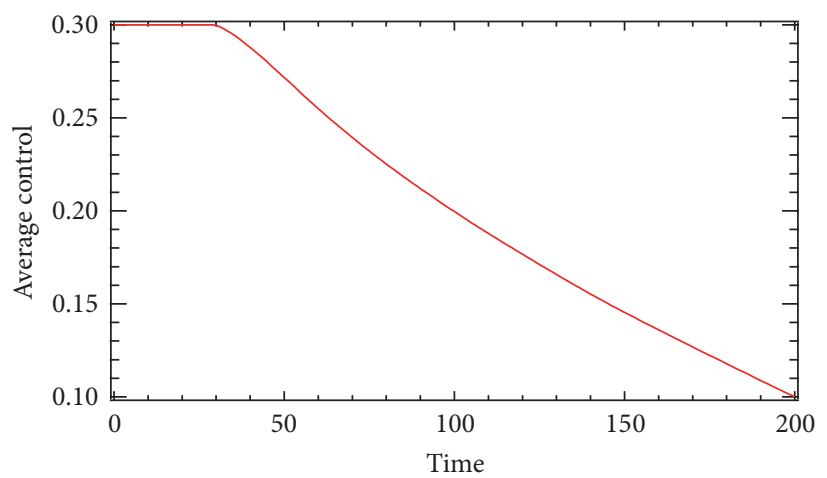

- Optimal control

(a)

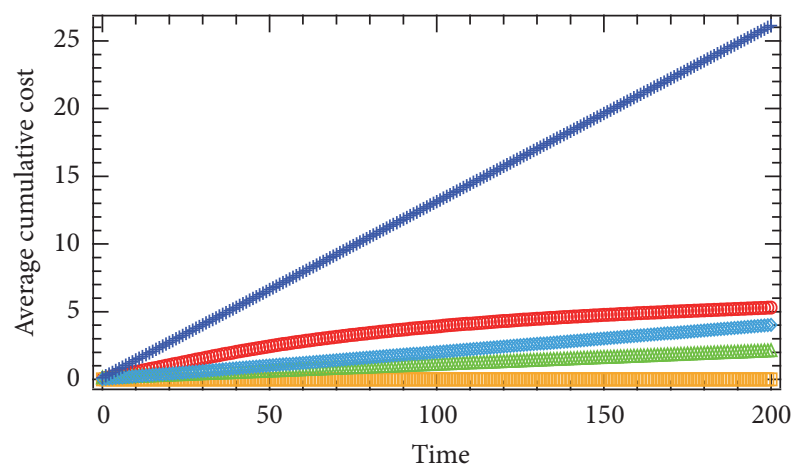

- Optimal control

$\square \gamma_{L}=0.0, \gamma_{B}=0.0$

$\Delta \gamma_{L}=0.0, \gamma_{B}=0.1$

$\diamond \gamma_{L}=0.1, \gamma_{B}=0.1$

$+\gamma_{L}=0.2, \gamma_{B}=0.3$

(c)

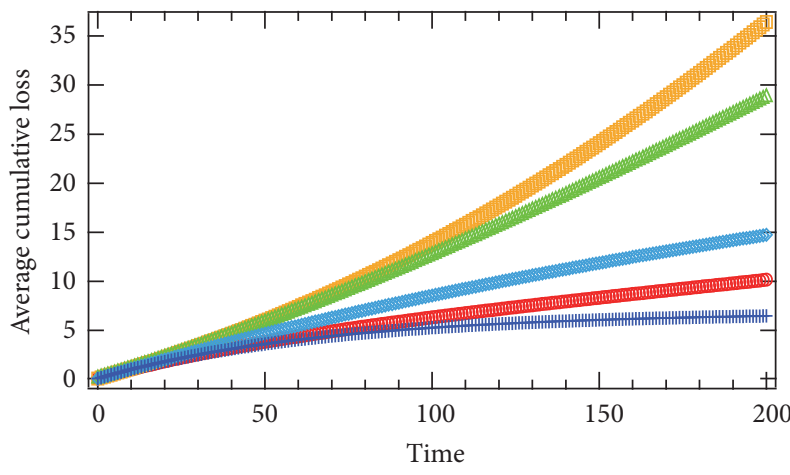

- Optimal control

$\square \gamma_{L}=0.0, \gamma_{B}=0.0$

$\triangle \gamma_{L}=0.0, \gamma_{B}=0.1$

$\diamond \gamma_{L}=0.1, \gamma_{B}=0.1$

$+\gamma_{L}=0.2, \gamma_{B}=0.3$

(b)

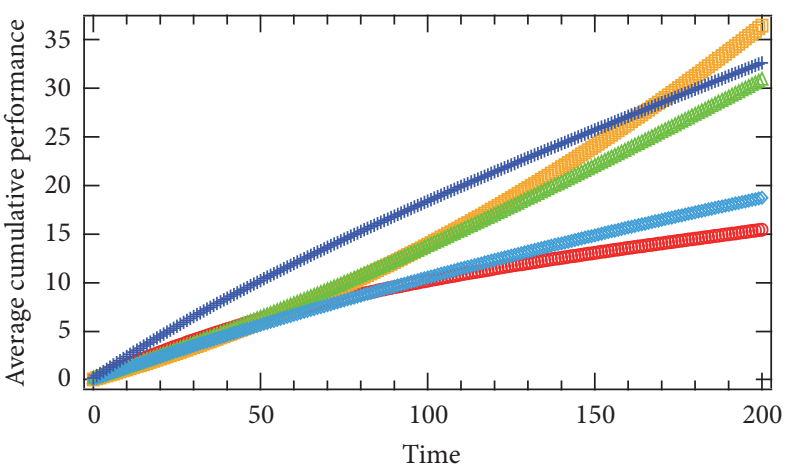

- Optimal control

$\square \gamma_{L}=0.0, \gamma_{B}=0.0$

$\triangle \gamma_{L}=0.0, \gamma_{B}=0.1$

$\diamond \gamma_{L}=0.1, \gamma_{B}=0.1$

$+\gamma_{L}=0.2, \gamma_{B}=0.3$

(d)

FIgure 4: (a) The AC functions, (b) the ACL functions, (c) the ACC functions, and (d) the ACP functions for the optimal dynamic control strategies in Example 12.

$$
\begin{aligned}
& \cdot \int_{0}^{t}\left\|\operatorname{diag}\left(L_{i}(s)-L_{i}^{*}(s)+B_{i}(s)-B_{i}^{*}(s)\right)\right\| \\
& \cdot\left\|\mathbf{L}^{*}(s)\right\| d s+\left\|\mathbf{A}_{B}\right\| \\
& \cdot \int_{0}^{t}\left\|\operatorname{diag}\left(L_{i}(s)-L_{i}^{*}(s)+B_{i}(s)-B_{i}^{*}(s)\right)\right\| \\
& \cdot\left\|\mathbf{B}^{*}(s)\right\| d s+\int_{0}^{t}\left\|\operatorname{diag}\left(\alpha_{i}+\gamma_{L, i}(s)\right)\right\| \\
& \cdot\left\|\mathbf{L}(s)-\mathbf{L}^{*}(s)\right\| d s \\
& +\int_{0}^{t}\left\|\operatorname{diag}\left(\gamma_{L, i}(s)-\gamma_{L, i}^{*}(s)\right)\right\| \cdot\left\|\mathbf{L}^{*}(s)\right\| d s \\
& \leq\left(2\left\|\mathbf{A}_{L}\right\|+2\left\|\mathbf{A}_{B}\right\|+\overline{\gamma_{L}}-\underline{\gamma_{L}}\right) t+\left(\left\|\mathbf{A}_{L}\right\|+\bar{\alpha}+\overline{\gamma_{L}}\right)
\end{aligned}
$$$$
\cdot \int_{0}^{t}\left\|\mathbf{L}(s)-\mathbf{L}^{*}(s)\right\| d s+\left\|\mathbf{A}_{B}\right\|
$$$$
\cdot \int_{0}^{t}\left\|\mathbf{B}(s)-\mathbf{B}^{*}(s)\right\| d s .
$$

As

$$
\begin{aligned}
\mathbf{B}(t)= & \mathbf{B}_{0}^{*}+\int_{0}^{t} \operatorname{diag}\left(\alpha_{i}\right) \mathbf{L}(s) d s \\
& -\int_{0}^{t} \operatorname{diag}\left(\gamma_{B, i}(s)\right) \mathbf{B}(s) d s, \\
\mathbf{B}^{*}(t)= & \mathbf{B}_{0}^{*}+\int_{0}^{t} \operatorname{diag}\left(\alpha_{i}\right) \mathbf{L}^{*}(s) d s \\
& -\int_{0}^{t} \operatorname{diag}\left(\gamma_{B, i}^{*}(s)\right) \cdot \mathbf{B}^{*}(s) d s,
\end{aligned}
$$


we get

$$
\begin{aligned}
\mathbf{B}(t) & -\mathbf{B}^{*}(t) \\
= & \int_{0}^{t} \operatorname{diag}\left(\alpha_{i}\right)\left[\mathbf{L}(s)-\mathbf{L}^{*}(s)\right] d s \\
& -\int_{0}^{t} \operatorname{diag}\left(\gamma_{B, i}(s)\right)\left[\mathbf{B}(s)-\mathbf{B}^{*}(s)\right] d s \\
& -\int_{0}^{t} \operatorname{diag}\left(\gamma_{B, i}(s)-\gamma_{B, i}^{*}(s)\right) \mathbf{B}^{*}(s) d s .
\end{aligned}
$$

Thus,

$$
\begin{aligned}
& \left\|\mathbf{B}(t)-\mathbf{B}^{*}(t)\right\| \\
& \leq \int_{0}^{t}\left\|\operatorname{diag}\left(\alpha_{i}\right)\right\| \cdot\left\|\mathbf{L}(s)-\mathbf{L}^{*}(s)\right\| d s \\
& \quad+\int_{0}^{t}\left\|\operatorname{diag}\left(\gamma_{B, i}(s)\right)\right\| \cdot\left\|\mathbf{B}(s)-\mathbf{B}^{*}(s)\right\| d s \\
& \quad+\int_{0}^{t}\left\|\operatorname{diag}\left(\gamma_{B, i}(s)-\gamma_{B, i}^{*}(s)\right)\right\| \cdot\left\|\mathbf{B}^{*}(s)\right\| d s \\
& \leq\left(\overline{\gamma_{B}}-\underline{\gamma_{B}}\right) t+\bar{\alpha} \int_{0}^{t}\left\|\mathbf{L}(s)-\mathbf{L}^{*}(s)\right\| d s \\
& \quad+\overline{\gamma_{B}} \int_{0}^{t}\left\|\mathbf{B}(s)-\mathbf{B}^{*}(s)\right\| d s .
\end{aligned}
$$

Applying the Gronwall inequality [47], we get

$$
\begin{aligned}
\left\|\mathbf{I}(t)-\mathbf{I}^{*}(t)\right\| & \leq c_{1} t+c_{1} c_{2} \int_{0}^{t} s e^{c_{2}(t-s)} d s \\
& =\frac{c_{1}}{c_{2}}\left(e^{c_{2} t}-1-c_{2} t\right) .
\end{aligned}
$$

Hence, we deduce that

$$
\begin{aligned}
& \left|J(\gamma(\cdot))-J\left(\gamma^{*}(\cdot)\right)\right| \\
& \leq \sum_{i=1}^{N} \int_{0}^{T}\left|L_{i}(t)-L_{i}^{*}(t)\right| d t \\
& +\sum_{i} \int_{0}^{T}\left|B_{i}(t)-B_{i}^{*}(t)\right| d t \\
& +\sum_{i} p_{i} \int_{0}^{T}\left|\gamma_{L, i}^{\theta}(t)-\gamma_{L, i}^{* \theta}(t)\right| d t \\
& +\sum_{i=1}^{N} q_{i} \int_{0}^{T}\left|\gamma_{B, i}^{\theta}(t)-\gamma_{B, i}^{* \theta}(t)\right| d t \\
& \leq 2 N \int_{0}^{T}\left\|\mathbf{I}(t)-\mathbf{I}^{*}(t)\right\| d t \\
& +\sum_{i=1}^{N} p_{i} \int_{0}^{T}\left|\gamma_{L, i}^{\theta}(t)-\gamma_{L, i}^{* \theta}(t)\right| d t \\
& +\sum_{i=1}^{N} q_{i} \int_{0}^{T}\left|\theta_{i}^{k}(t)-\theta_{i}^{* k}(t)\right| d t \\
& \leq \frac{2 N c_{1}}{c_{2}}\left(e^{c_{2} T}-1-c_{2} T-\frac{c_{2}^{2} T^{2}}{2}\right) \\
& +\sum_{i=1}^{N} p_{i} \int_{0}^{T}\left|\gamma_{L, i}^{\theta}(t)-\gamma_{L, i}^{* \theta}(t)\right| d t \\
& +\sum_{i=1}^{N} q_{i} \int_{0}^{T}\left|\gamma_{B, i}^{\theta}(t)-\gamma_{B, i}^{* \theta}(t)\right| d t .
\end{aligned}
$$

Although this estimation is rough, it takes the first step towards the accurate performance evaluation of actual dynamic control strategies of disruptive computer viruses.

\section{Conclusions and Remarks}

This paper has studied the problem of containing disruptive computer viruses in a cost-effective way. The problem has been modeled as an optimal control problem. A criterion for the existence of an optimal control has been given, and the optimality system has been derived. Some examples of the optimal dynamic control strategy have been presented. Finally, the performance of an actual control strategy of disruptive viruses has been estimated.

Towards this direction, there are a number of problems that are worth studying. First, the bandwidth resources consumed in the virus control process should be measured and incorporated in the cost. Second, the optimal dynamic control problem should be investigated under sophisticated epidemic models such as the impulsive epidemic models $[51,52]$, the stochastic epidemic models [53-55], and the epidemic models on time-varying networks [56-58]. Last, 
it is rewarding to apply the methodology developed in this paper to the optimal dynamic control of rumor spreading [59-61].

\section{Conflicts of Interest}

The authors declare that there are no conflicts of interest regarding the publication of this paper.

\section{Acknowledgments}

This work is supported by Natural Science Foundation of China (Grant nos. 61572006, 71301177), National SciTech Support Plan (Grant no. 2015BAF05B03), Basic and Advanced Research Program of Chongqing (Grant no. cstc2013jcyjA1658), and Fundamental Research Funds for the Central Universities (Grant no. 106112014CDJZR008823).

\section{References}

[1] P. Szor, The Art of Computer Virus Research and Defense, Addison-Wesley Education Publishers, 2005.

[2] Y. Wang, S. Wen, Y. Xiang, and W. Zhou, "Modeling the propagation of worms in networks: a survey," IEEE Communications Surveys and Tutorials, vol. 16, no. 2, pp. 942-960, 2014.

[3] J. O. Kephart and S. R. White, "Directed-graph epidemiological models of computer viruses," in Proceedings of the IEEE Computer Society Symposium on Research in Security and Privacy, pp. 343-359, Oakland, Calif, USA, May 1991.

[4] J. O. Kephart and S. R. White, "Measuring and modeling computer virus prevalence," in Proceedings of the IEEE Computer Society Symposium on Research in Security and Privacy, pp. 2-15, Oakland, Calif, USA, May 1993.

[5] J. R. Piqueira and V. O. Araujo, "A modified epidemiological model for computer viruses," Applied Mathematics and Computation, vol. 213, no. 2, pp. 355-360, 2009.

[6] B. K. Mishra and S. K. Pandey, "Dynamic model of worms with vertical transmission in computer network," Applied Mathematics and Computation, vol. 217, no. 21, pp. 8438-8446, 2011.

[7] Y. Yao, W. Xiang, A. Qu, G. Yu, and F. Gao, "Hopf bifurcation in an SEIDQV worm propagation model with quarantine strategy," Discrete Dynamics in Nature and Society, vol. 2012, Article ID 304868, 18 pages, 2012.

[8] L. Feng, X. Liao, Q. Han, and L. Song, "Modeling and analysis of peer-to-peer botnets," Discrete Dynamics in Nature and Society, vol. 2012, Article ID 865075, 18 pages, 2012.

[9] Y. Li, J. Pan, L. Song, and Z. Jin, "The influence of user protection behaviors on the control of internet worm propagation," Abstract and Applied Analysis, vol. 2013, Article ID 531781, 13 pages, 2013.

[10] Y. Yao, X.-W. Xie, H. Guo, G. Yu, F.-X. Gao, and X.-J. Tong, "Hopf bifurcation in an Internet worm propagation model with time delay in quarantine," Mathematical and Computer Modelling, vol. 57, no. 11-12, pp. 2635-2646, 2013.

[11] Y. Muroya, Y. Enatsu, and H. Li, "Global stability of a delayed SIRS computer virus propagation model," International Journal of Computer Mathematics, vol. 91, no. 3, pp. 347-367, 2014.

[12] Z. Zhang and H. Yang, "Hopf bifurcation of an SIQR computer virus model with time delay," Discrete Dynamics in Nature and Society, vol. 2015, Article ID 101874, 8 pages, 2015.
[13] R. Pastor-Satorras and A. Vespignani, "Epidemic spreading in scale-free networks," Physical Review Letters, vol. 86, no. 14, pp. 3200-3203, 2001.

[14] M. Barthélemy, A. Barrat, R. Pastor-Satorras, and A. Vespignani, "Velocity and hierarchical spread of epidemic outbreaks in scale-free networks," Physical Review Letters, vol. 92, no. 17, Article ID 178701, 2004.

[15] M. Yang, G. Chen, and X. Fu, "A modified SIS model with an infective medium on complex networks and its global stability," Physica A: Statistical Mechanics and Its Applications, vol. 390, no. 12, pp. 2408-2413, 2011.

[16] J. Ren, Y. Xu, and J. Liu, "Investigation of dynamics of a virusantivirus model in complex network," Physica A: Statistical Mechanics and its Applications, vol. 421, pp. 533-540, 2015.

[17] J. Ren, J. Liu, and Y. Xu, "Modeling the dynamics of a networkbased model of virus attacks on targeted resources," Communications in Nonlinear Science and Numerical Simulation, vol. 31, no. 1-3, pp. 1-10, 2016.

[18] P. Van Mieghem, J. Omic, and R. Kooij, "Virus spread in networks," IEEE/ACM Transactions on Networking, vol. 17, no. 1, pp. 1-14, 2009.

[19] P. Van Mieghem, "The $N$-intertwined SIS epidemic network model," Computing, vol. 93, no. 2, pp. 147-169, 2011.

[20] S. Xu, W. Lu, and Z. Zhan, "A stochastic model of multivirus dynamics," IEEE Transactions on Dependable and Secure Computing, vol. 9, no. 1, pp. 30-45, 2012.

[21] F. D. Sahneh, F. N. Chowdhury, and C. M. Scoglio, "On the existence of a threshold for preventive behavioral responses to suppress epidemic spreading," Scientific Reports, vol. 2, article 632, 2012.

[22] P. Van Mieghem, "Approximate formula and bounds for the time-varying susceptible-infected-susceptible prevalence in networks," Physical Review E-Statistical, Nonlinear, and Soft Matter Physics, vol. 93, no. 5, Article ID 052312, 2016.

[23] C. Nowzari, V. M. Preciado, and G. J. Pappas, "Analysis and control of epidemics: a survey of spreading processes on complex networks," IEEE Control Systems, vol. 36, no. 1, pp. 2646, 2016.

[24] V. M. Preciado, F. D. Sahneh, and C. Scoglio, "A convex framework for optimal investment on disease awareness in social networks," in Proceedings of the 1st IEEE Global Conference on Signal and Information Processing (GlobalSIP '13), pp. 851-854, Austin, Tex, USA, December 2013.

[25] V. M. Preciado, M. Zargham, C. Enyioha, A. Jadbabaie, and G. J. Pappas, "Optimal resource allocation for network protection against spreading processes," IEEE Transactions on Control of Network Systems, vol. 1, no. 1, pp. 99-108, 2014.

[26] C. Nowzari, M. Ogura, V. M. Preciado, and G. J. Pappas, "Optimal resource allocation for containing epidemics on time-varying networks," in Proceedings of the 49th Asilomar Conference on Signals, Systems and Computers (ACSSC '15), pp. 1333-1337, Pacific Grove, Calif, USA, November 2015.

[27] H. Shakeri, F. D. Sahneh, C. Scoglio, P. Poggi-Corradini, and V. M. Preciado, "Optimal information dissemination strategy to promote preventive behaviors in multilayer epidemic networks," Mathematical Biosciences and Engineering. MBE, vol. 12, no. 3, pp. 609-623, 2015.

[28] N. J. Watkins, C. Nowzari, V. M. Preciado, and G. J. Pappas, "Optimal resource allocation for competitive spreading processes on bilayer networks," IEEE Transactions on Control of Network Systems, 2016. 
[29] E. Hansen and T. Day, "Optimal control of epidemics with limited resources," Journal of Mathematical Biology, vol. 62, no. 3, pp. 423-451, 2011.

[30] M. H. R. Khouzani, S. Sarkar, and E. Altman, "Optimal dissemination of security patches in mobile wireless networks," IEEE Transactions on Information Theory, vol. 58, no. 7, pp. 4714-4732, 2012.

[31] S. Eshghi, M. H. R. Khouzani, S. Sarkar, and S. S. Venkatesh, "Optimal patching in clustered malware epidemics," IEEE/ACM Transactions on Networking, vol. 24, no. 1, pp. 283-298, 2016.

[32] A. Khanafer and T. Basar, "An optimal control problem over infected networks," in Proceedings of the International Conference of Control, Dynamic Systems, and Robotics, Ottawa, Canada, May 2014.

[33] L.-X. Yang, M. Draief, and X. Yang, "The optimal dynamic immunization under a controlled heterogeneous node-based SIRS model," Physica A. Statistical Mechanics and Its Applications, vol. 450, pp. 403-415, 2016.

[34] L.-X. Yang and X. Yang, "Propagation behavior of virus codes in the situation that infected computers are connected to the internet with positive probability," Discrete Dynamics in Nature and Society, vol. 2012, Article ID 693695, 13 pages, 2012.

[35] L.-X. Yang and X. Yang, "The spread of computer viruses under the influence of removable storage devices," Applied Mathematics and Computation, vol. 219, no. 8, pp. 3914-3922, 2012.

[36] L.-X. Yang, X. Yang, L. Wen, and J. Liu, "A novel computer virus propagation model and its dynamics," International Journal of Computer Mathematics, vol. 89, no. 17, pp. 2307-2314, 2012.

[37] L.-X. Yang, X. Yang, Q. Zhu, and L. Wen, "A computer virus model with graded cure rates," Nonlinear Analysis: Real World Applications, vol. 14, no. 1, pp. 414-422, 2013.

[38] Y. Muroya and T. Kuniya, "Global stability of nonresident computer virus models," Mathematical Methods in the Applied Sciences, vol. 38, no. 2, pp. 281-295, 2015.

[39] M. Yang, Z. Zhang, Q. Li, and G. Zhang, "An SLBRS model with vertical transmission of computer virus over the Internet," Discrete Dynamics in Nature and Society, vol. 2012, Article ID 925648, 17 pages, 2012.

[40] L.-X. Yang, X. Yang, J. Liu, Q. Zhu, and C. Gan, "Epidemics of computer viruses: a complex-network approach," Applied Mathematics and Computation, vol. 219, no. 16, pp. 8705-8717, 2013.

[41] L.-X. Yang and X. Yang, "A new epidemic model of computer viruses," Communications in Nonlinear Science and Numerical Simulation, vol. 19, no. 6, pp. 1935-1944, 2014.

[42] C. Zhang, W. Liu, J. Xiao, and Y. Zhao, "Hopf bifurcation of an improved SLBS model under the influence of latent period," Mathematical Problems in Engineering, vol. 2013, Article ID 196214, 10 pages, 2013.

[43] L.-X. Yang and X. Yang, "The impact of nonlinear infection rate on the spread of computer virus," Nonlinear Dynamics. An International Journal of Nonlinear Dynamics and Chaos in Engineering Systems, vol. 82, no. 1-2, pp. 85-95, 2015.

[44] L. Chen, K. Hattaf, and J. Sun, "Optimal control of a delayed SLBS computer virus model," Physica A. Statistical Mechanics and Its Applications, vol. 427, pp. 244-250, 2015.

[45] L.-X. Yang, M. Draief, and X. Yang, "The impact of the network topology on the viral prevalence: a node-based approach," PLoS ONE, vol. 10, no. 7, Article ID e0134507, 2015.
[46] D. Liberzon, Calculus of Variations and Optimal Control Theory, Princeton University Press, Princeton, NJ, USA, 2012.

[47] R. C. Robinson, An Introduction to Dynamical Systems: Continuous and Discrete, Pearson Prentice Hall, Upper Saddle River, NJ, USA, 2005.

[48] A.-L. Barabási and R. Albert, "Emergence of scaling in random networks," Science, vol. 286, no. 5439, pp. 509-512, 1999.

[49] D. J. Watts and S. H. Strogatz, "Collective dynamics of 'smallworld' networks," Nature, vol. 393, no. 6684, pp. 440-442, 1998.

[50] https://snap.stanford.edu/data/egonets-Facebook.html.

[51] Y. Yao, L. Guo, H. Guo, G. Yu, F.-X. Gao, and X.-J. Tong, "Pulse quarantine strategy of internet worm propagation: modeling and analysis," Computers and Electrical Engineering, vol. 38, no. 5, pp. 1047-1061, 2012.

[52] Y. Yao, X. Feng, W. Yang, W. Xiang, and F. Gao, "Analysis of a delayed Internet worm propagation model with impulsive quarantine strategy," Mathematical Problems in Engineering, vol. 2014, Article ID 369360, 18 pages, 2014.

[53] T. Britton, "Stochastic epidemic models: a survey," Mathematical Biosciences, vol. 225, no. 1, pp. 24-35, 2010.

[54] J. Amador and J. R. Artalejo, "Stochastic modeling of computer virus spreading with warning signals," Journal of the Franklin Institute. Engineering and Applied Mathematics, vol. 350, no. 5, pp. 1112-1138, 2013.

[55] J. Amador, "The stochastic SIRA model for computer viruses," Applied Mathematics and Computation, vol. 232, pp. 1112-1124, 2014.

[56] Y. Schwarzkopf, A. Rakos, and D. Mukamel, "Epidemic spreading in evolving networks," Physical Review E, vol. 82, no. 3, pp. 336-354, 2010.

[57] E. Valdano, L. Ferreri, C. Poletto, and V. Colizza, "Analytical computation of the epidemic threshold on temporal networks," Physical Review X, vol. 5, no. 2, Article ID 021005, 2015.

[58] M. Ogura and V. M. Preciado, "Stability of spreading processes over time-varying large-scale networks," IEEE Transactions on Network Science and Engineering, vol. 3, no. 1, pp. 44-57, 2016.

[59] J. R. Piqueira, "Rumor propagation model: An Equilibrium Study," Mathematical Problems in Engineering, vol. 2010, Article ID 631357, 7 pages, 2010.

[60] S. Nizamani, N. Memon, and S. Galam, "From public outrage to the burst of public violence: an epidemic-like model," Physica A: Statistical Mechanics and Its Applications, vol. 416, pp. 620-630, 2014.

[61] J. Xu, M. Zhang, and J. Ni, "A coupled model for government communication and rumor spreading in emergencies," Advances in Difference Equations, vol. 2016, article 208, 2016. 


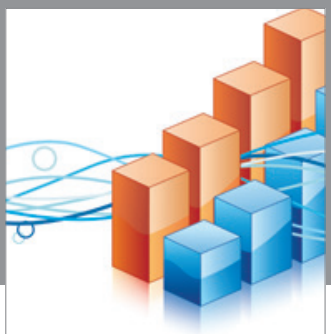

Advances in

Operations Research

vatem alat4

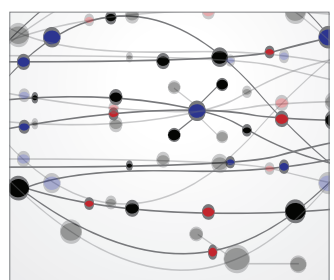

\section{The Scientific} World Journal
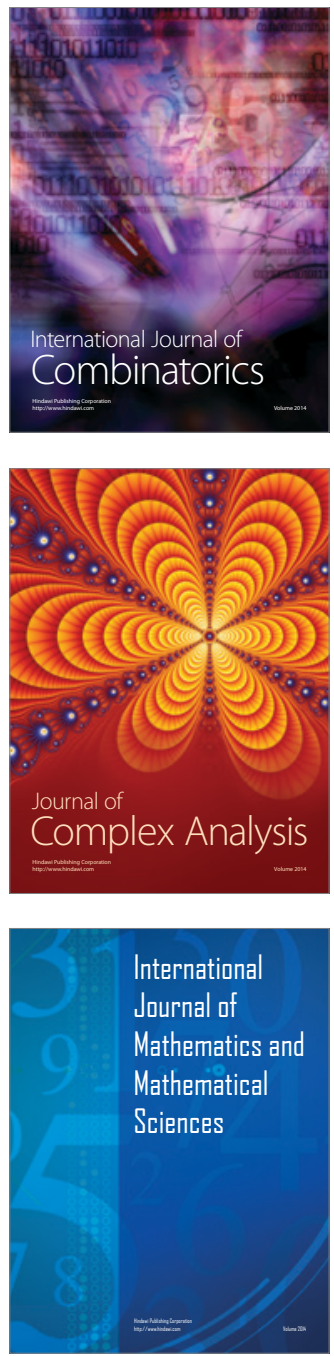
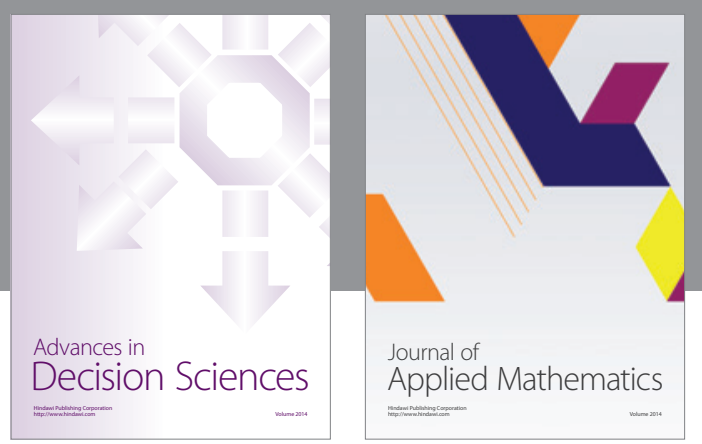

Algebra

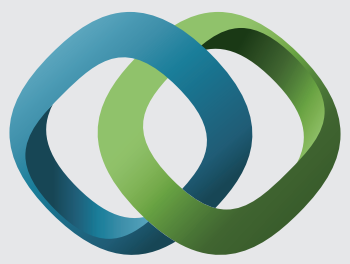

\section{Hindawi}

Submit your manuscripts at

https://www.hindawi.com
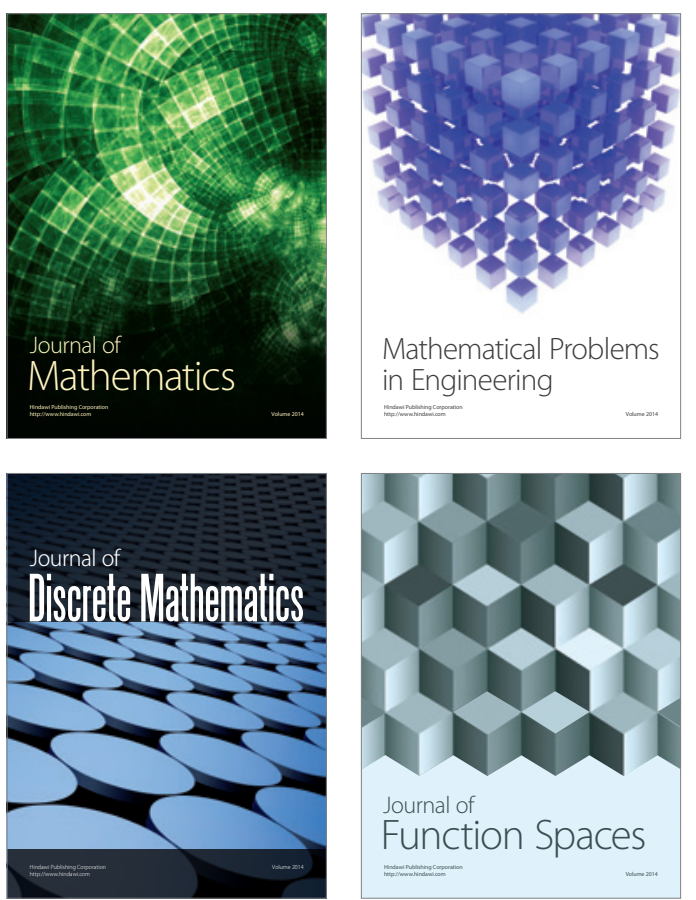

Mathematical Problems in Engineering
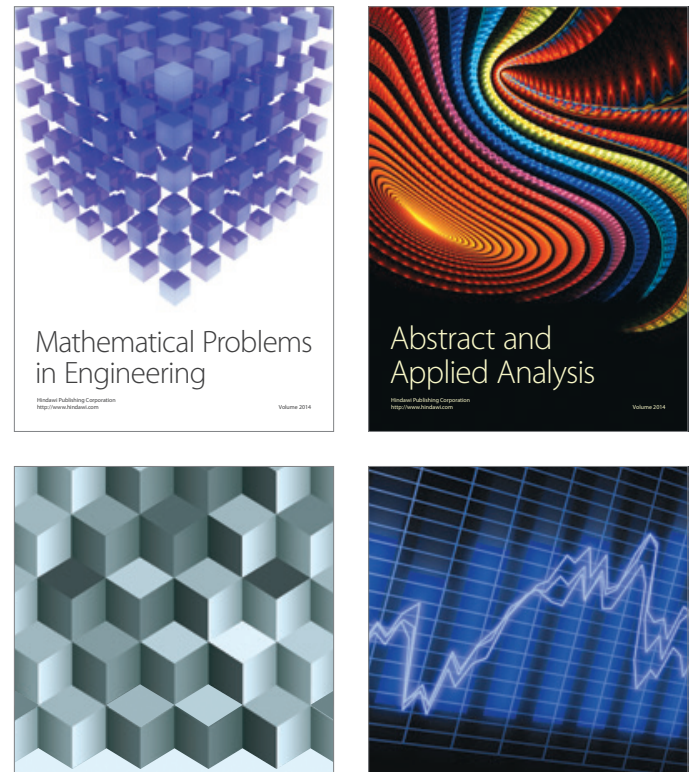

Journal of

Function Spaces

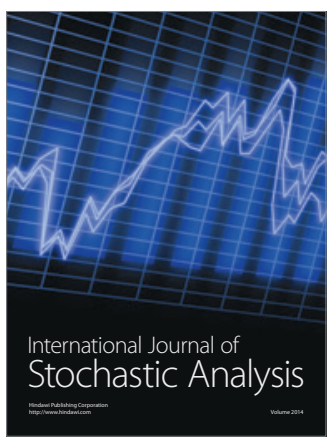

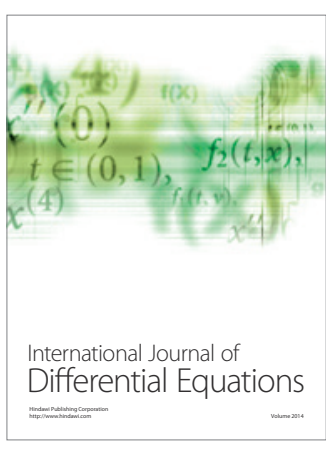
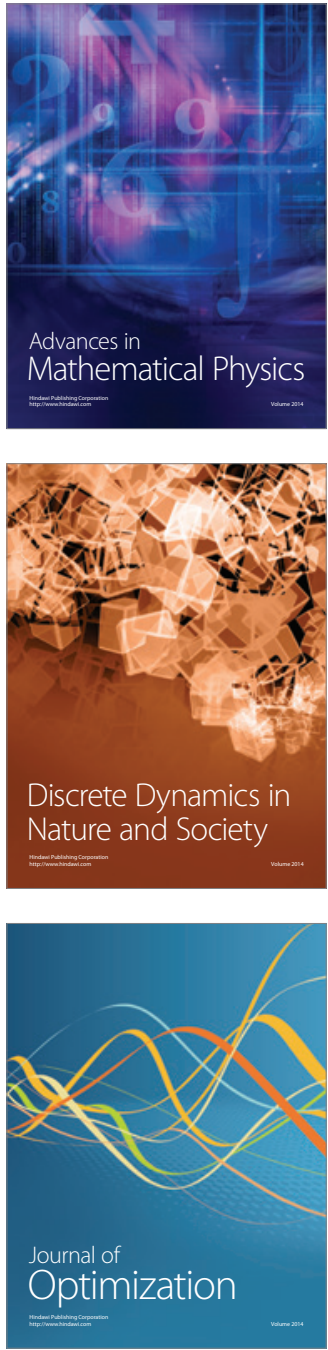